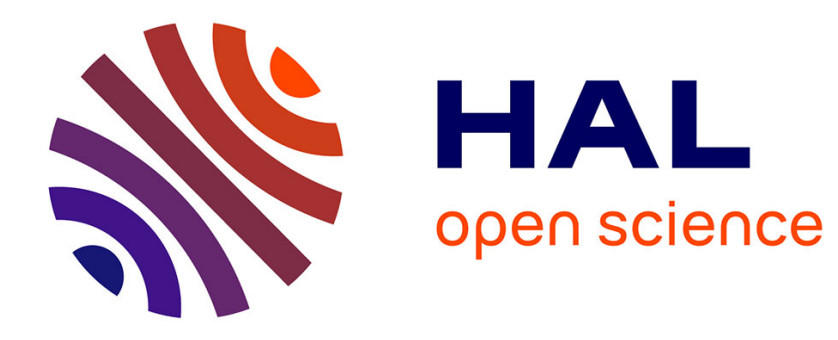

\title{
Enquête sur les modes d'existence des êtres mathématiques
}

Guy Wallet, Stefan Neuwirth

\section{To cite this version:}

Guy Wallet, Stefan Neuwirth. Enquête sur les modes d'existence des êtres mathématiques. Philosophia Scientiae, 2019, 23-3, pp.83-108. 10.4000/philosophiascientiae.2080 . hal-01943079v2

\section{HAL Id: hal-01943079 \\ https://hal.science/hal-01943079v2}

Submitted on 2 Aug 2020

HAL is a multi-disciplinary open access archive for the deposit and dissemination of scientific research documents, whether they are published or not. The documents may come from teaching and research institutions in France or abroad, or from public or private research centers.
L'archive ouverte pluridisciplinaire HAL, est destinée au dépôt et à la diffusion de documents scientifiques de niveau recherche, publiés ou non, émanant des établissements d'enseignement et de recherche français ou étrangers, des laboratoires publics ou privés. 


\title{
Enquête sur les modes d'existence des êtres mathématiques
}

\author{
Guy Wallet
}

Stefan Neuwirth

\begin{abstract}
Résumé
L'objet de cet essai est l'accueil des entités mathématiques dans l'architecture des modes d'existence proposée par Bruno Latour dans le cadre de son ontologie pluraliste du monde moderne [Latour 2012]. Les travaux de Reviel Netz sur l'émergence des mathématiques grecques [Netz 1999] et de Charles Sanders Peirce sur la dimension diagrammatique de l'activité mathématique [Peirce 1933-1958], [Peirce 1976] sont employés pour proposer une réponse dans le cadre d'une conception empirique des mathématiques basée sur la notion d'expérience chère à William James [James 2005] et inspirée par certains aspects de la philosophie de Per Martin-Löf [Martin-Löf 1987]. Cette approche permet de penser la solide certitude dont la démonstration dote les résultats mathématiques tout en invalidant son interprétation comme la marque d'un accès direct à une vérité absolue et transcendante.
\end{abstract}

\begin{abstract}
This essay inquires how mathematical beings could be inserted into the architecture of modes of existence proposed by Bruno Latour in the framework of his pluralist and renewed ontology of the modern world [Latour 2012]. An answer to this question is put forward with the aid of the work of Reviel Netz on the emergence of Greek mathematics [Netz 1999], and of Charles Sanders Peirce on the diagrammatic dimension of mathematical practice [Peirce 1933-1958], [Peirce 1976], in the framework of an empiric conception of mathematics based on the notion of experience according to William James [James 2005], and inspired by certain aspects of Per Martin-Löf's philosophy [Martin-Löf 1987]. It provides a way of describing the firm certainty with which proofs endow theorems, while invalidating the interpretation of this certainty as the mark of a direct access to an absolute and transcendental truth.
\end{abstract}




\section{Introduction}

Désignée par l'abréviation EME dans la suite de ce texte, l'Enquête sur les modes d'existence est à la fois le livre [Latour 2012] et le site participatif [Latour 2013]. Ils proposent de revisiter le cœur de notre modernité - sciences, techniques, droit, religion, politique, économie, entre autres - et d'élaborer une description explicite d'une multiplicité de formes d'existence, chacune dotée de son propre régime de vérité. Ce projet d'une ontologie pluraliste s'oppose au point de vue qui ne reconnait qu'une seule forme d'existence, à savoir celle qui relève de la Science et plus largement de la Raison. Il ne s'agit pas de critiquer l'institution scientifique, ni de nier son importance, mais de mettre la science à sa juste place en restituant finement la spécificité de son fonctionnement tout en concevant la possibilité d'autres institutions et d'autres formes d'existence. L'un des motifs de cette refonte ontologique est de fournir un cadre approprié pour penser et réagir face à la menace d'une catastrophe écologique et climatique de plus en plus pressante.

Un moment essentiel dans la genèse de l'EME est la mise en évidence du mode d'existence prépondérant dans la démarche scientifique pour produire une connaissance objective, avant tout dans les sciences expérimentales et de terrain : c'est le mode de la référence, caractérisé par la construction de chaines de référence. Les caractéristiques principales de ce mode et des chaines de référence seront brièvement rappelées au début de la partie 5.1. Il suffit pour l'instant de savoir qu'une chaine de référence est une succession d'inscriptions scripto-visuelles ${ }^{1}$, chacune étant produite à partir de la précédente. La chaine s'arrête lorsque l'inscription obtenue est suffisamment claire et explicite pour éclairer le problème étudié. Quelque chose existe objectivement lorsqu'une chaine de référence montre cette chose.

Les mathématiques, composante du champ scientifique, relèvent-elles de ce mode d'existence? Cette question n'a pas de réponse évidente et n'est pas franchement abordée dans l'état actuel de l'EME. En première analyse, on peut préciser deux traits principaux de cette difficulté.

- Ranger les mathématiques dans le mode de la référence nécessite de montrer comment l'activité mathématique relève elle aussi des chaines de référence. Comme la caractéristique essentielle de la pratique des mathématiques est l'élaboration de démonstrations, il semble nécessaire d'établir un pont entre la notion de chaine de référence et celle de démonstration, ce qui ne va pas de soi. Cette difficulté est liée au fait que les chaines de référence ont été mises en évidence dans la pratique des sciences expérimentales, pratique assurément fort différente de celle qui prévaut dans une science formelle

1. La description dans l'Eme du mode de la référence fait usage du terme d'inscription pour désigner un dispositif concret qui permet d'opérer une transition entre une face plus matérielle et une face qui relève plus de l'écriture ou du calcul : «par exemple une vitrine d'exposition sur laquelle on pose un spécimen est une inscription au même titre que l'écran d'un scanner médical ou un tableau excel » [Latour 2013, « Livre », colonne « Vocabulaire », entrée « Inscription »]. 
comme les mathématiques.

- La clarification de ce problème se heurte à la captation philosophique, vieille comme les mathématiques et la philosophie grecques, qui fait de la démonstration mathématique la forme paradigmatique de la démarche rationnelle, capable de mener directement et sans perte à la vérité pensée comme absolue et indépendante des acteurs humains (voir [Netz 1999, p. 290] et [Latour 2009, p. 189-191]). Cette conception de la démonstration rend inimaginable tout rapprochement substantiel avec la notion de chaine de référence décrite dans l'EME.

Et pourtant, Bruno Latour a depuis longtemps émis l'hypothèse que le cas des sciences formelles et abstraites comme les mathématiques devrait pouvoir se traiter de manière plus évidente que celui des sciences de laboratoire ou de terrain (voir [Latour 1985]). Il suppose que c'est le poids de nos préjugés philosophiques, particulièrement prégnants en ce qui concerne les mathématiques, qui nous aveugle sur ce point. Le point de vue développé dans la suite corrobore cette intuition.

Notre ambition est de déterminer un mode d'existence pour les mathématiques qui soit compatible à la fois avec l'EME et avec l'expérience pratique des mathématiciens. Le titre du présent texte évoque les modes d'existence au pluriel parce que notre enquête identifie un autre mode, qui fait intervenir le calcul mécanique de l'ordinateur comme étape essentielle d'un raisonnement; il utilise l'article déterminé, « les modes », pour afficher notre ambition d'une enquête ouverte et la plus large possible.

Nous nous attaquerons d'abord à l'arrière-fond philosophique évoqué en proposant une conception empirique de la notion de démonstration mathématique. Cette proposition est basée sur la notion d'expérience chère à William James [James 2005], mais elle est aussi inspirée de la philosophie de Per Martin-Löf [Martin-Löf 1987] sans oublier de précieuses réflexions de Ludwig Wittgenstein [Wittgenstein 2006] et John Dewey [Dewey 2014]. Il résulte de notre approche que chaque résultat mathématique est doté de la certitude résultant de sa démonstration, tout en étant affecté d'une forme de fragilité irréductible héritée du fait que la démonstration est d'abord une expérience. Cela invalide l'interprétation de cette certitude comme la marque d'un accès direct à une vérité absolue et transcendante.

Cette étude préparatoire mais essentielle menée, nous en déduirons, somme toute assez simplement, un mode d'existence propre aux êtres mathématiques. Celui-ci respecte les caractéristiques d'un mode d'existence selon l'EME. De plus, il présente à la fois des analogies significatives et quelques différences notables avec le mode d'existence de la référence. La question de l'articulation des deux peut alors commencer à être discutée.

Le présent article est une version condensée mais autosuffisante de l'étude plus détaillée [Wallet \& Neuwirth 2018]. 


\section{L'apport de Netz et de Peirce : la place des diagrammes en mathématiques}

\subsection{L'émergence des mathématiques grecques selon Netz}

Dans [Latour 2009], Bruno Latour présente un ouvrage qui constitue selon lui la première avancée décisive dans la direction d'une « approche non formaliste du formalisme ». Il s'agit de The shaping of deduction in Greek mathematics : a study in cognitive history, [Netz 1999]; il se place en opposition explicite avec la démarche épistémologique classique de Thomas Kuhn [Kuhn 1972], qui s'intéresse aux systèmes de croyances partagées - les paradigmes - sur lesquels se fonderait le développement des connaissances scientifiques, ainsi qu'au basculement de ces paradigmes lors des révolutions scientifiques. Netz met en doute que le travail scientifique se fonde sur ces paradigmes qui apparaissent plutôt après coup dans les explications des épistémologues. Au contraire, ce sont pour lui les pratiques partagées qui constituent la base efficiente du développement concret des sciences : il propose de mettre en lumière celles qui révèlent les ressources cognitives spécifiques aux mathématiques grecques.

La première pratique abordée par Netz est celle de l'introduction du diagramme avec lettres qui est l'outil emblématique des mathématiques grecques (figure 1). C'est un dispositif qui, en relation d'interdépendance avec le texte pur, offre une vue synoptique du cas considéré et joue le rôle d'univers de référence du texte. Cet univers est doublement fini : les objets qui sont marqués sont bornés et en nombre fini. La nature des objets représentés sur le diagramme n'est pas précisée et la question de la réalité ultime des objets mathématiques n'est pas posée : l'ambigüité ontologique parait constitutive de cette discipline. Ce parti pris a pour effet bénéfique d'écarter les questions qui fâchent, celles pour lesquelles un consensus est quasiment impossible, particulièrement dans la tradition de la polémique propre à la démocratie grecque.

La seconde pratique relevée par Netz est l'utilisation d'un langage fortement spécifique. Sa première propriété est la petite taille de son lexique : 143 mots constituent $95 \%$ du corpus des œuvres d'Archimède, L'Arénaire mise à part. Un univers lexical suffisant et commode pour le travail de démonstration est ainsi extrait de la vaste étendue de la langue grecque. La deuxième propriété de ce langage est sa structure remarquable : il procède par combinaison d'un petit nombre de groupes de mots qui se répètent avec fort peu de variabilité. La présence massive de ces « formules » indique qu'elles ont un rôle cognitif important dans la structuration et la communication des argumentaires.

Selon Netz, ces deux pratiques sont les fondements de l'émergence et de l'efficience du mode déductif dans les mathématiques grecques. 


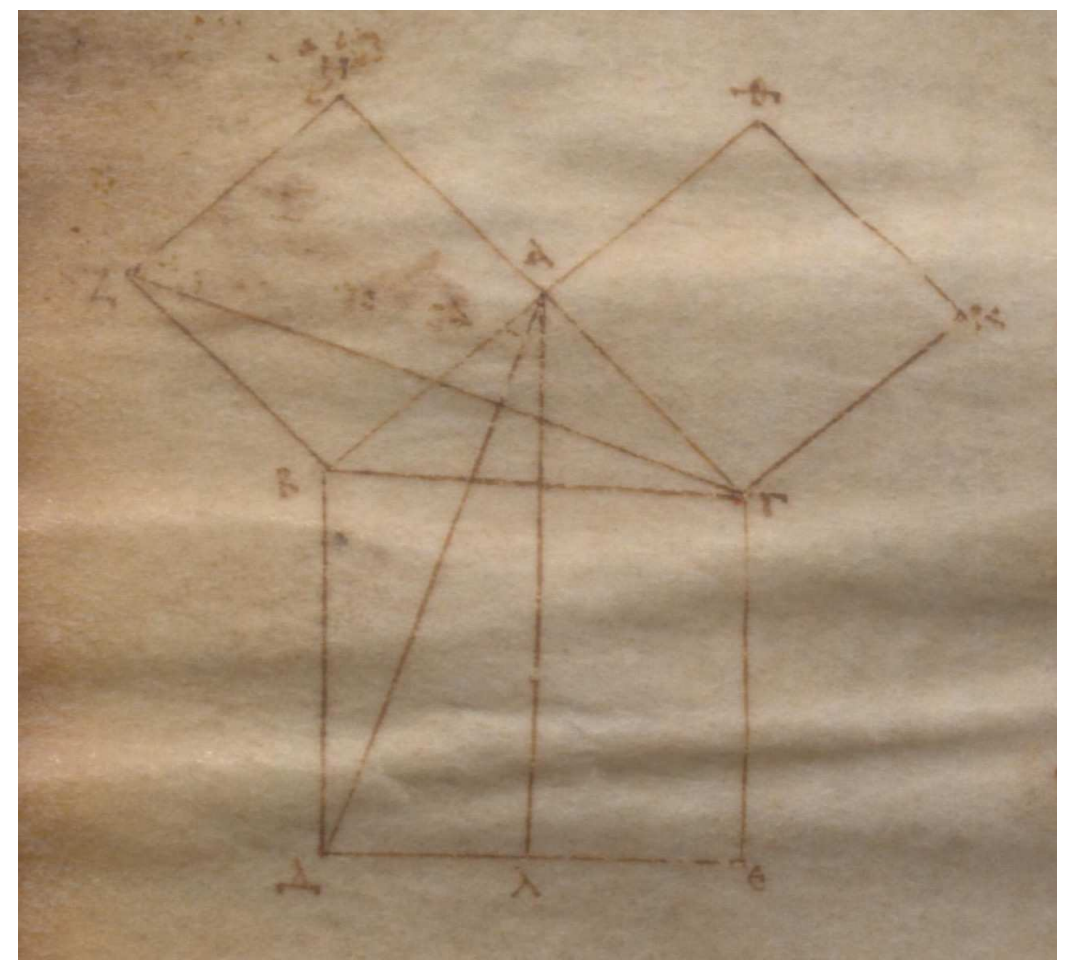

FiguRE 1 - Le diagramme avec lettres qui accompagne le théorème de Pythagore dans les Éléments d'Euclide sur le folio 31 du manuscrit MS D'Orville 301 de l'an 888, reproduit avec l'aimable autorisation de l'Institut de mathématiques Clay.

\subsection{Le diagramme au cour des mathématiques selon C. S. Peirce}

Cette partie s'appuie sur les travaux [Chauviré 1987], [Chauviré 2008].

Le point de vue de Peirce sur les mathématiques se fonde sur sa sémiotique. En toute généralité, un signe désigne quelque chose qui représente une autre chose. Peirce n'impose pas au signe d'être simple ou minimal : les signes qu'il considère sont souvent des agencements complexes. Par exemple, un diagramme avec lettres de la géométrie grecque est un signe au sens de Peirce au même titre qu'une simple lettre nommant un objet géométrique dans le même diagramme.

Il considère que la pensée nécessite l'utilisation des signes au sens fort suivant : la fonction des signes n'est pas de représenter une pensée déjà produite par un processus se déroulant ailleurs; au contraire, les signes constituent le substrat indispensable à la constitution et au développement de la pensée et ultimement, l'acte 
de penser consiste à manipuler des signes. De plus, Peirce classe les signes en trois catégories, à savoir le symbole, l'indice et l'icône, en fonction du rapport qu'un signe entretient avec son objet. Le symbole est un signe de nature arbitraire dont la seule relation avec la chose représentée est une convention : la lettre nommant un objet géométrique est un symbole. L'indice est un signe qui entretient un rapport physique ou matériel avec la chose représentée : le mouvement de rotation des ailes d'un moulin est un indice du vent. L'icône est un signe qui a un rapport de ressemblance avec la chose qu'il représente : le dessin d'un arbre est une icône de la notion d'arbre.

Dans la pratique de la géométrie, le diagramme tracé explicitement sur un support n'est pas le véritable objet de l'étude. La meilleure preuve en est que des imperfections dans le tracé sont tolérées. Comme ce diagramme tient lieu de la configuration géométrique idéale sur laquelle porte le raisonnement, il est un signe au sens de Peirce. Il n'est pas difficile d'identifier la catégorie de ce signe puisque le symbole et l'indice sont d'emblée disqualifiés. Le diagramme géométrique est donc une icône : sa vertu est de représenter une configuration géométrique par un rapport de ressemblance. D'après Peirce, cette ressemblance porte sur la forme de la configuration, sachant que pour lui, la forme d'une chose est la structure de cette chose, c'est-à-dire l'ensemble des relations entre les parties constitutives de cette chose. Il est notable que de nombreux secteurs des mathématiques utilisent explicitement des diagrammes de nature géométrique alors même que les objets étudiés ne relèvent pas de la géométrie. Pour ne donner qu'un exemple emblématique, il suffit de considérer la théorie des catégories (figure 2). Toutes ces formes

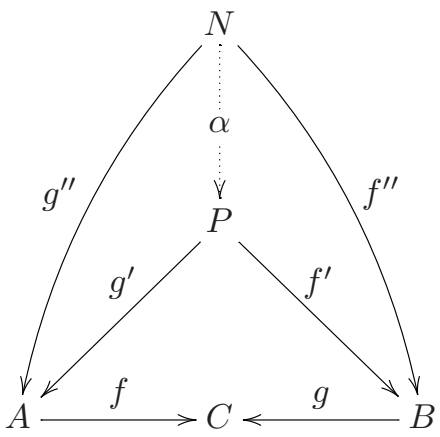

Figure 2 - Le diagramme de la propriété universelle du produit fibré $\left(P, g^{\prime}, f^{\prime}\right)$ de deux morphismes $f: A \rightarrow C$ et $g: B \rightarrow C$.

géométrisées de diagrammes relèvent clairement de l'analyse précédente.

Peirce introduit alors une extension de la notion de diagramme qui rompt le lien avec la figure géométrique. En toute généralité, un diagramme est une icône 
qui met en scène de manière perceptible la forme de la chose représentée ${ }^{2}$.

Cette extension permet à Peirce d'énoncer sa thèse fondamentale sur la sémiologie des mathématiques : dans tous ses champs d'étude, c'est l'essence même de la pensée mathématique en acte de se traduire constamment par un travail de construction, d'observation et de transformation de diagrammes. Cette thèse prend le contre-pied de l'idée reçue selon laquelle le mathématicien accède directement aux formes ou concepts abstraits. Le point de vue de Peirce englobe aussi une interprétation éclairante de l'intuition mathématique en accord avec cette thèse : l'intuition d'une forme abstraite est la saisie empirique de cette forme sur une icône qui la représente.

Pour comprendre la pertinence de ce point de vue, on peut le tester sur une formule algébrique extraite au hasard des Éléments de mathématique de Bourbaki [Bourbaki 1970, p. A.I.93].

$$
\left(\sum_{\lambda \in \mathrm{L}} x_{\lambda}\right)^{n}=\sum_{|\beta|=n} \frac{n !}{\prod_{\lambda \in \mathrm{L}} \beta_{\lambda} !} \prod_{\lambda \in \mathrm{L}} x_{\lambda}^{\beta_{\lambda}} .
$$

Dans le droit fil de l'analyse de Peirce, une formule de ce type, avec ses notations et sa manière d'occuper l'espace sur le support de l'écriture - savoir-faire inventé graduellement au cours du développement historique de ce domaine - est aussi un diagramme. En effet, cette graphie donne à voir au mathématicien qui l'observe nombre de propriétés de l'objet abstrait ainsi représenté, et prépare les opérations qu'on pourra lui appliquer. C'est ainsi qu'il est possible d'interpréter la pratique de l'algèbre comme un travail d'observation et de transformation de diagrammes.

À ce degré de généralité, un fragment de langage peut être porteur d'une dimension diagrammatique. Précisément, une séquence purement textuelle dans un développement mathématique constitue un diagramme, sous réserve qu'elle se révèle susceptible de mettre en scène une forme abstraite de manière perceptible. Dans cet ordre d'idée, la structure en « formules » du texte mathématique grec est interprétable comme l'explicitation d'une dimension diagrammatique de ce texte. En général, séquences textuelles, formules mathématiques et éventuellement figures géométriques sont inséparables et concourent à la constitution de diagrammes composés qui sont des mixtes de ces divers ingrédients.

2. En plus du diagramme, l'analyse percienne distingue deux autres types d'icônes : l'image, qui désigne un signe perçu comme la représentation analogique de quelque chose - l'image, c'est « la ressemblance à l'état pur », le « degré zéro de l'icône»-, et la métaphore, qui consiste à remplacer une relation sémiotique par une nouvelle relation sémiotique sur la base d'une similarité non préexistante introduite à cette occasion [Verhaegen 1994] . Quel que soit l'intérêt de ces notions par ailleurs fort discutées dans la littérature [Verhaegen 1994], [Esquenazi 1997], [Lefebvre 2013], c'est le diagramme dans l'acception généralisée précédente qui constitue pour Peirce le processus sémiotique essentiel à l'œuvre dans les mathématiques. C'est cette thèse qui est retenue dans le présent article, et elle constitue l'un des appuis à la conception empirique de la démonstration donnée dans la partie 4 . 


\subsection{Le point sur la question d'un mode d'existence en mathématiques après Netz et Peirce}

Les travaux de Netz et de Peirce diffèrent au point qu'il peut sembler aventureux de vouloir en tirer une leçon commune. Et pourtant, ces deux approches se complètent relativement bien en ce qui concerne la place du diagramme dans les mathématiques. En effet, le diagramme géométrique avec lettres dont Netz a analysé l'importance dans les mathématiques grecques est généralisé par Peirce en un dispositif iconique jouant un rôle essentiel dans l'ensemble des mathématiques. D'autre part, Netz a mis en évidence des facteurs linguistiques spécifiques chez les Grecs, facteurs dont on peut admettre la persistance jusqu'à nos jours et qui constituent un cadre dans lequel la notion de diagramme généralisé au sens de Peirce se révèle pertinente pour l'activité mathématique en général.

La description qu'ils donnent des mathématiques est fortement corrélée avec l'analyse anthropologique développée par l'EME. C'est vrai en ce qui concerne l'étude concrète du fonctionnement réel d'une science et l'importance donnée aux véritables technologies intellectuelles que sont le travail sur les diagrammes couplé avec un langage enrégimenté et la fabrication et l'étude des inscriptions scriptovisuelles. Dans les deux cas, il s'agit de « rematérialiser » la connaissance mathématique, c'est-à-dire de montrer en quoi elle dépend de procédés concrets de construction et de manipulation d'inscriptions, dont l'importance est généralement ignorée par l'approche purement épistémique du savoir mathématique.

Après Netz et Peirce, que peut-on dire alors sur la manière dont se manifestent les êtres mathématiques? Ce sont les « existants » [Latour 2013, « Livre », colonne 《Vocabulaire », entrée 《Êtres, existants»] qui résultent des agencements particuliers produits par les mathématiciens. On y voit se répéter la forme suivante : l'introduction d'un cadre de travail par un jeu de définitions, de diagrammes, de notations et de commentaires, puis l'annonce du résultat à démontrer suivie de sa validation par une démonstration proprement dite. La leçon de Netz et de Peirce est que ces formes ne deviennent productives et partageables par une communauté que sous certaines conditions techniques et cognitives. Marquées par l'usage des démonstrations comme moyen de validation, ce sont ces mathématiques qui se sont trouvées au cœur de la révolution scientifique et sont maintenant pratiquées dans les lieux de recherche partout dans le monde.

Ceci étant admis, la place centrale des démonstrations dans les mathématiques suggère que les êtres mathématiques manifestent leur existence par des parcours en relation avec les démonstrations dans lesquelles ils sont impliqués. Est-il possible d'interpréter ces parcours démonstratifs comme des chaines de référence au sens de l'EME? Peut-on concevoir les démonstrations comme les trajectoires d'un mode d'existence spécifique aux entités mathématiques? Pour répondre à ces questions, nous commençons par un long détour pour élaborer une conception non formaliste des mathématiques. 


\section{La démonstration et l'idée formaliste de sa perfection}

La logique contemporaine met à disposition plusieurs types de description de la notion de démonstration captant plus ou moins bien la forme de la pratique argumentaire des mathématiciens, dont le calcul nommé « déduction naturelle »; la description qui suit s'en inspire. Introduit par le mathématicien et logicien allemand Gerhard Gentzen [Gentzen 1955], il a pour objet premier de proposer un formalisme reproduisant le mode naturel du raisonnement mathématique par opposition à la méthode axiomatique de Russell et Whitehead : le raisonnement mathématique naturel repose non pas sur la référence à une poignée d'axiomes mais sur l'application de règles d'inférences claires, efficaces et en aussi grand nombre que nécessaire dans chaque secteur de cette discipline.

Selon la terminologie utilisée dans les travaux de Martin-Löf [Martin-Löf 1987], un résultat mathématique dont on sait qu'on a le droit de l'affirmer est un jugement. Un jugement est donc un élément établi de connaissance mathématique.

La forme la plus simple d'un jugement est « $A$ est vrai », où $A$ désigne une proposition. Par exemple, l'énoncé que « la somme des angles d'un triangle est égale à deux angles droits » est une proposition, et l'affirmation que cette proposition est vraie est un jugement qui est par exemple établi par la démonstration donnée dans les Éléments d'Euclide (livre I, proposition 32).

De quel droit peut-on affirmer un jugement? Certains sont donnés préalablement à la phase déductive; ils traduisent une sorte d'évidence basique et immédiate relative aux objets considérés et au cadre de travail. Les autres jugements sont ceux qu'on peut obtenir par déduction, c'est-à-dire par application de règles d'inférence : chacune de ces règles décrit comment des jugements permettent d'établir un autre jugement. Ces règles d'inférence reprennent sous une forme épurée les bases de la logique du raisonnement humain. La déduction naturelle représente une démonstration mathématique comme un enchainement de jugements ${ }^{3}$, chaque jugement étant établi à partir des précédents par une règle d'inférence, le dernier jugement établi étant l'affirmation du théorème.

Il n'y a pas grand-chose à reprocher à la description de la notion de démonstration développée ci-dessus sinon qu'elle est excessivement brève et formelle. Toutefois, ce mode de description donne prise à une interprétation formaliste, version moderne de l'antique captation philosophique des mathématiques grecques.

En effet, on a vu qu'une démonstration apparait comme une structure constituée de jugements reliés par des inférences logiques; or les inférences logiques sont parfaitement caractérisées et cataloguées par le travail des logiciens depuis

3. Une démonstration peut être un peu plus complexe qu'une simple suite organisée comme un parcours linéaire et se présenter comme un arbre de preuve. Cette même structure serait appropriée pour les chaines de référence. 
le siècle dernier : cette structure peut donc être simulée par un calcul en réduisant le texte de la démonstration à une série de symboles assemblés selon des règles syntaxiques précises, sans utiliser la langue naturelle. La démonstration apparait ainsi comme un dispositif formel objectif dont la validité repose sur son objectivité, c'est-à-dire sur son caractère indépendant de l'approche subjective de l'esprit humain. En conséquence, une fois qu'elle est convenablement formalisée, une démonstration mathématique constitue une forme d'argumentation imparable, incontestable, menant inéluctablement à sa conclusion. Ainsi, la vérité dévoilée par une démonstration acquiert un caractère universel, immuable et permanent.

Pour une défense de ce point de vue, nous renvoyons à l'introduction du livre I des Éléments de mathématique de Bourbaki [Bourbaki 1954], qui a pour but explicite de montrer les changements que le formalisme apporte à la notion de démonstration.

Ainsi, la mise au clair de ce qu'est une démonstration peut conforter l'idée de la perfection absolue de l'argumentation mathématique. Non seulement cette conception mérite d'être questionnée, mais aussi le glissement insidieux qui fait de la démonstration mathématique le modèle par excellence de la méthode rationnelle permettant, selon « les modernes ${ }^{4} »$, l'accès direct et complet à la Vérité quel que soit le domaine considéré.

\section{Une conception empirique du jugement mathématique}

\subsection{L'expérience d'un jugement}

Pour échapper au biais qu'un jugement serait obtenu grâce au dispositif formel que constitue une démonstration formalisée, indépendamment des acteurs mathématiciens concernés, de leur appréhension de ladite démonstration, de leurs tentatives, de leurs discussions, de leurs calculs, il suffit de remarquer qu'une configuration de signes ne devient une démonstration - formelle ou pas - qu'à la condition que des acteurs humains puissent en faire l'expérience. Cette remarque nous met sur la voie d'une autre approche philosophique dans l'esprit de l'empirisme radical de James [James 2005]. Cette doctrine est centrée sur la notion d'expérience, c'est-à-dire tout ce dont un sujet individuel fait l'expérience dans le flux continu de sa propre vie. Autrement dit, l'expérience est le champ instantané du présent, à la fois continu et changeant, qui constitue le vécu de chaque personne. L'intérêt

4. Conformément à l'utilisation de ce terme dans l'Eme [Latour 2013, « Livre», colonne « Vocabulaire», entrée « Modernes, modernisation»], les modernes adhèrent pleinement au grand récit de l'émancipation selon lequel, depuis la révolution scientifique, s'établirait progressivement mais irréversiblement la distinction absolue entre Illusion et Raison, entre un passé archaïque et un futur émancipé, entre Croyance et Savoir Vrai. Voir [Latour 2012] pour de plus amples développements sur ce thème et son rapport avec la crise écologique. 
de l'empirisme jamesien pour notre questionnement se manifeste dans le principe suivant qui en offre un résumé percutant :

N'admettre que ce dont on puisse faire l'expérience, et rendre justice à tout ce qui peut être objet d'expérience. [James 2005, préface.]

De ce point de vue, un jugement est une expérience d'un certain type qu'il faut maintenant essayer de décrire. Qu'est-ce qui caractérise les jugements parmi les expériences porteuses de connaissance? La réponse qui va être donnée est inspirée par la lecture du travail [Martin-Löf 1987] de Martin-Löf, dont l'intuitionnisme n'est pas totalement étranger à l'empirisme, comme relevé dans [Bourdeau 2004].

La propriété fondamentale qui fait qu'un énoncé mathématique est un jugement est qu'on sait qu'on a le droit de l'affirmer : on en a une démonstration. La divergence avec le point de vue formaliste se marque si l'on insiste sur la nature de ce savoir en mettant en avant la notion d'expérience. C'est ce qui est fait avec la définition suivante.

Une expérience de démonstration d'un jugement est une expérience par laquelle un mathématicien saisit l'évidence de ce jugement.

On peut dire qu'une expérience de démonstration est une expérience par laquelle ce mathématicien voit ce jugement; alors, pour le sujet de cette expérience, le jugement est, pleinement, sans restriction au point qu'il lui est impensable d'en douter, de s'y soustraire. Chaque terme de la définition a son importance : « expérience » bien entendu pour qualifier un segment du flux de la vie propre à un individu, "mathématicien » pour indiquer que la reconnaissance pleine et entière de cette expérience nécessite une compétence généralement acquise par l'enseignement et la pratique au sein d'une communauté scientifique spécifique, enfin « l'évidence » pour marquer qu'une expérience de démonstration rend visible et manifeste le jugement, qu'elle expose au regard la totalité d'un arrangement qui le valide sans conteste et l'établit comme fait mathématique. Dans l'utilisation qui vient d'en être faite, les expressions « voir un jugement », « rendre visible » ou « exposer au regard $»$ ne sont pas de simples métaphores destinées à désigner la saisie directe par l'esprit de propriétés abstraites. Comme l'a montré Peirce, la dimension diagrammatique des mathématiques permet au mathématicien d'amener dans sa perception sensible la forme de la configuration abstraite dont il traite, et il peut ainsi l'observer et la manipuler avec la force brute que donne cette perception.

Cette expérience est en partie un acte mental, mais elle ne se réduit pas au moment ultime pendant lequel le mathématicien est saisi par l'évidence. Ce moment est préparé par la démarche souvent laborieuse d'analyse critique et d'appropriation à laquelle se livre le mathématicien en composant ou en décryptant une inscription faite de textes, de figures, de schémas, de formules, de calculs, etc. Ce n'est qu'à la fin de cette phase que l'expérience peut surgir pleine et entière. C'est la totalité du processus menant à l'évidence finale qui est l'expérience de démonstration. 
Contrairement à l'approche formaliste de la démonstration, une expérience de démonstration d'un jugement ne se fonde pas sur l'utilisation explicite de techniques et méthodes logiques, mais sur la mise en place d'un dispositif menant à la manifestation incontestable du bien-fondé de ce jugement. Dans l'ordre conceptuel, celle-ci précède l'analyse logique du raisonnement, qui elle procède d'une mise en forme postérieure à l'expérience de démonstration. Le travail de construction d'une expérience de démonstration consiste à fabriquer un cheminement souvent indirect, permettant de transporter étape par étape l'évidence jusqu'à obtenir l'évidence finale du jugement. Ceci produit à chaque étape une expérience de démonstration d'un jugement intermédiaire. Ainsi une expérience de démonstration d'un jugement se scinde en une succession d'expériences de jugements intermédiaires, c'est-à-dire en une succession d'expériences de l'évidence de chacun de ces jugements.

L'évidence se propage de jugement en jugement pour culminer par l'évidence du jugement final. Il est bon de noter que ce n'est pas une force qui caractérise la certitude accordée à un jugement, c'est plutôt une qualité, celle qui résulte du cheminement de l'évidence qui aboutit en pleine lumière, sans zone d'ombre et sans délégation à un oracle ${ }^{5}$, au jugement considéré.

Nous proposons de lire dans ce sens le passage suivant du Continu de Hermann Weyl.

Le célèbre ouvrage de Dedekind, Nature et fonction du nombre, 1888, préf. $1^{\text {re }}$ éd., s'ouvre sur cette phrase : « Ce qui est démontrable ne doit pas, en science, être cru sans démonstration ». Cette assertion est probablement typique de la pensée de la plupart des mathématiciens, elle n'en prend pas moins les choses à l'envers. Comme si un rapport de fondation aussi médiat que celui que nous nommons démonstration, était capable de susciter une « croyance » sans que nous nous assurions de l'exactitude de chaque pas isolé au moyen d'une intuition immédiate! C'est cette intuition (non pas la démonstration), qui est partout la source ultime de la connaissance, elle consiste à avoir « l'expérience directe du vrai ${ }^{6} »$. [Weyl 1994, p. 59.]

\subsection{Inscription de démonstration et partage d'un jugement}

Maintenant qu'a été introduit le concept d'expérience de démonstration, il est notable qu'il ne recouvre pas l'idée usuelle de démonstration. En effet, dans un texte mathématique, le passage censé justifier un théorème est lui-même qualifié

5. Il y a délégation à un oracle lorsque le cheminement de l'évidence est rompu et remplacé localement par le recours à un moyen externe à l'expérience de l'évidence. Par exemple, ce moyen peut être l'avis d'un expert assurant d'autorité que tel énoncé est valide, ou bien encore le recours à un calcul sur machine approprié.

6. Weyl cite ici Edmund Husserl : « Une évidence n'est, au contraire, rien d'autre que "l'expérience vécue" de la vérité » [Husserl 1959, § 51]. 
de démonstration (ou de preuve) du théorème. Or il est clair que cet objet concret est distinct de l'expérience de démonstration du théorème en question. Un mathématicien sait que, généralement, la simple lecture d'une démonstration d'un théorème est loin de suffire pour en éprouver l'évidence. La définition qui suit précise cette distinction.

Une inscription de démonstration d'un jugement donné est une inscription scripto-visuelle (couchée sur le papier, tracée sur un tableau, enregistrée dans un fichier informatique...) présentant une configuration d'arguments textuels et graphiques susceptible de permettre l'expérience de démonstration du jugement.

La notion d'inscription de démonstration d'un jugement est donc intrinsèquement différente de celle d'expérience de démonstration puisque la première est faite de textes, de tableaux, de schémas, de diagrammes, et que la seconde est une expérience se concluant chez celui qui l'éprouve par l'acte mental de l'évidence du jugement.

Une inscription de démonstration d'un jugement joue le rôle d'un guide pour le cheminement de l'évidence jusqu'à l'expérience de démonstration de ce jugement. C'est un peu le même rapport qui existe entre le topo-guide décrivant une randonnée (bel exemple d'inscription scripto-visuelle) et l'accomplissement sur le terrain de cette même randonnée (expérience éventuellement délicate et incertaine). L'idée habituelle de démonstration se voit scindée en deux notions différentes mais complémentaires, l'inscription de démonstration et l'expérience de démonstration.

L'intérêt principal d'une inscription de démonstration d'un jugement est de conserver et de communiquer des éléments permettant de valider ce jugement. L'acte de communiquer la démonstration d'un jugement est essentiel dans la vie scientifique de la communauté des mathématiciens. Cet acte présuppose la croyance que la démontrabilité d'un jugement est une notion partageable.

Pourtant, il va de soi qu'une expérience de démonstration d'un jugement est essentiellement privée du fait que l'expérience éprouvée par un individu lui appartient en tant que moment de sa vie propre et que toute autre personne est dans l'incapacité d'y accéder pleinement. Néanmoins, on constate dans la pratique de la communauté des mathématiciens que la validation d'un jugement est une notion partageable : ceux-ci se mettent d'accord pour reconnaitre qu'une inscription de démonstration permet de valider un jugement, et ceci signifie qu'un travail d'exploration de ce document génère chez eux une expérience de démonstration du jugement.

Avec Wittgenstein, nous pouvons estimer que cette propriété ne relève pas d'une explication philosophique mais d'un arrière-fond de pratiques et de formes de vie [Wittgenstein 2004] transmis par l'enseignement de cette science, dans lequel on apprend collectivement à éprouver l'évidence de tel ou tel énoncé : l'apprentissage des mathématiques est aussi un apprentissage collectif de la notion de démontra- 
bilité et du consensus qui en découle.

Lorsqu'un jugement est partagé par un nombre significatif d'acteurs compétents sur le sujet, la communauté des mathématiciens accorde sa confiance à ce jugement. Cela implique que ce résultat devient une sorte de « fait mathématique » sur lequel tout mathématicien peut s'appuyer sans être dans l'obligation d'en éprouver lui-même l'évidence. Cette manière de procéder peut sembler être une forme de recours à un oracle, mais elle en diffère parce que d'une part il y a eu partage de la démonstration par un panel d'experts de confiance, et que d'autre part il est toujours possible à quiconque de se reporter à la démonstration. Quoi qu'il en soit, l'acceptation d'un nouveau jugement se fait en deux étapes, l'étape individuelle ou quasi-individuelle suivie de l'étape collective, ce qui renforce considérablement la fiabilité du résultat démontré. Ce mécanisme de validation collectif ne doit pas occulter le fait que c'est l'expérience de démonstration qui constitue la pierre angulaire et la spécificité de l'activité mathématique, même lorsque cette expérience est déléguée à un groupe d'experts.

\subsection{Le phénomène des erreurs post demonstrationem}

Contrairement à ce qui advient dans la vie courante, les raisonnements mathématiques ont la réputation d'être d'une solidité infaillible et d'imposer un consensus. Elle est justifiée par la nature même de la validation d'un jugement mathématique, explicitée dans la partie 4.2, qui constitue un réquisit d'une très grande exigence. Néanmoins, l'idée d'un discours parfait résulte d'un glissement abusif. Une manifestation claire de cet abus de réputation réside dans le phénomène des erreurs post demonstrationem (terminologie introduite par les auteurs du présent texte). Le caractère irrépressible de ce phénomène a été remarqué par Martin-Löf [Martin-Löf 1987]. Il y a erreur post demonstrationem lorsque : 1) à un moment donné, un jugement est validé par la procédure décrite précédemment ; 2 ) à un moment ultérieur, éventuellement longtemps après, une erreur est découverte dans cette validation. La manifestation de cette erreur est la découverte d'un argument nouveau dont l'effet concret est de rendre impossible l'expérience de l'évidence de ce jugement. Habituellement, la présence de ce type d'erreur est analysée comme la simple marque de l'imperfection ou de la finitude de l'esprit humain. Il en découle que les conséquences philosophiques du phénomène des erreurs post demonstrationem ne sont pas correctement perçues.

Il est vrai qu'on est face à un paradoxe. D'une part, le processus de la démonstration - expérience de l'évidence, inscription guidant cette expérience, partage de l'évidence guidée par l'inscription - est agencé pour exclure les erreurs : mener à son terme ce processus est la méthode, initiée par les mathématiciens grecs, qui apporte la garantie que le raisonnement ainsi produit est valide, exempt de toute faute. Mais d'autre part, la nature même de cette procédure ne permet pas d'exclure définitivement la possibilité qu'une erreur puisse être détectée par la suite. 
La raison en est qu'il n'y a aucun moyen extérieur à l'expérience de démonstration d'un jugement qui puisse permettre de s'assurer définitivement de sa validité. La fiabilité d'une démonstration se fonde uniquement sur une forme d'expérience humaine, celle de l'évidence d'un jugement.

D'une certaine manière, c'est la croyance que la technique de la démonstration n'est qu'un moyen pour accéder à une instance extérieure à cette expérience qui fonde l'idée de l'incontestabilité du raisonnement mathématique. Pour les philosophes platoniciens, cette instance était le monde transcendant des idées pures. Pour les modernes d'aujourd'hui, cette instance est plus volontiers le monde des objets formels. D'où cet ultime refuge pour la thèse de l'infaillibilité du discours mathématique : un raisonnement formalisé, c'est-à-dire complètement coulé dans le moule syntaxique de l'une des théories formalisant la pratique des mathématiques, peut sembler par essence complètement imparable. Si on admet cette thèse, on est tenté de voir tout raisonnement usuel comme une approximation nécessairement imparfaite d'un objet formel parfait, ce qui expliquerait à la fois la présence d'erreurs cachées dans la pratique ordinaire des mathématiques et une stratégie pour les éviter, à savoir celle de s'approcher autant que faire se peut de la formalisation complète. Mais qu'est-ce qu'une démonstration formelle? Comme cela a déjà été noté, une configuration de signes sur un support quelconque ne peut prendre le statut de démonstration formelle que dans le cas où elle est identifiée comme telle dans le cadre d'une expérience d'un sujet mathématicien. Quelle que soit la nature de cette expérience, il ne s'agit que d'une simple expérience humaine, inapte à fonder une infaillibilité sans restriction. En conséquence, la stratégie consistant à assoir la perfection absolue d'un raisonnement mathématique en le formalisant complètement ne peut pas fonctionner : cela ne fait que déplacer la possible intrusion future d'erreurs dans l'expérience qui assure que l'entité syntaxique obtenue possède la qualité de démonstration formelle.

\subsection{Deux remarques}

1. Jusqu'à ce point, l'analyse présentée auparavant n'a nullement pris en compte les différences, pratiques et théoriques, qui scindent la communauté des mathématiciens en deux écoles, d'une part les mathématiques classiques, et d'autre part les mathématiques constructives. En fait, la présentation d'une conception non formaliste de la démonstration présentée ci-haut s'applique de manière égale aux mathématiques développées dans ces deux groupes. Cela peut paraitre étonnant dans la mesure où ces deux courants s'opposent en particulier sur ce que doit être une démonstration. De plus, la définition de la notion d'expérience de démonstration donnée dans la partie 4.1 semble relativement proche des conceptions philosophiques développées dans l'intuitionnisme. Cependant, les auteurs du présent texte défendent que c'est la forme paradigmatique de la notion de démonstration, telle qu'elle est pratiquée depuis les Grecs, qui est captée par la définition choisie 
ici. Tout mathématicien, classique ou constructiviste, admet la validité d'une démonstration lorsque, au terme d'un travail d'exploration et de maturation, il est saisi par l'évidence de la conclusion. C'est dans le choix des moyens utilisés pour édifier la démonstration que l'opposition est pertinente. Par exemple, l'utilisation de la règle du tiers exclu est jugée légitime par un mathématicien classique au sens où elle lui semble marquée par l'évidence, alors que cette utilisation est rejetée par un mathématicien constructiviste pour qui ce même moyen rompt le cheminement de l'évidence.

2. Qu'en est-il de l'utilisation de calculs sur machine dans une démonstration? De manière analogue à ce qui se passe dans toutes les autres sciences, les progrès technologiques et théoriques font apparaitre des dispositifs de calcul susceptibles d'être utilisés dans la vérification ou la construction de démonstrations. C'est le cas des assistants de preuve comme Coq [The Coq development team 1984-2019]. Un exemple maintenant classique de la réussite de cette démarche est le théorème des quatre couleurs dont la démonstration complètement certifiée a été obtenue en 2004 [Gonthier 2005] et dont on ne possède aucune démonstration pouvant se passer de calculs sur machine.

Pour illustrer l'impact de l'usage des ordinateurs par un exemple simple, imaginons d'abord que la seule démonstration que nous aurions de ce que la somme des nombres entiers de 1 à 2000000 fait 2000001000000 était l'inscription du calcul de cette somme sur une machine; or nous avons une démonstration, qui est que cette somme est aussi celle des nombres entiers de 2000000 à 1, et qu'en additionnant terme à terme les termes de ces deux sommes, on obtient $2000000 \times 2000$ 001, qu'il reste à diviser par deux. L'évidence de cette démonstration est d'une autre qualité que l'inscription du calcul.

La question est de savoir en quoi la notion de démonstration présentée auparavant pourrait être modifiée par ces nouvelles pratiques. Par exemple, considérons une démonstration dont une partie n'est validée que par un calcul sur machine; est-ce qu'elle peut générer une démonstration au sens de l'expérience de l'évidence de son résultat? Il semble que non parce que le cheminement de l'évidence guidé par la démonstration est rompu : la partie de la démonstration prise en charge par la machine se présente comme une béance, du moins pour celui qui n'est pas capable de comprendre ce que fait le programme informatique. Notons que l'intrusion de la matérialité dans l'efficience des mathématiques n'est pas nouvelle et elle est même structurelle d'après la contribution de Netz sur l'émergence des mathématiques dans le monde grec; ce qui semble nouveau avec la mécanisation des démonstrations est que le dispositif mis en œuvre certes peut apporter une réponse, mais que cette dernière n'est pas de nature à favoriser la démonstration au sens de l'expérience de l'évidence de sa conclusion.

Néanmoins, un mathématicien peut être en capacité de saisir le fonctionnement de la machine, d'en démontrer la correction, pour finalement estimer que, tant bien 
que mal, l'évidence poursuit son chemin dans la partie traitée machinellement. Encore faut-il avoir une confiance absolue en le dispositif électronique piloté par le programme qui effectue la tâche demandée, de l'ordre de celle qu'on accorde à un résultat mathématique validé par une démonstration, c'est-à-dire une confiance qui exclut totalement le doute du fait qu'on est pénétré de son évidence par le contrôle qu'on a pu exercer sur l'intégralité du processus qui le valide. On voit bien que cela ne peut pas fonctionner ainsi. Dans le cas considéré, le détail du fonctionnement intime du processus mécanique qui valide le résultat n'est justement pas accessible : on est dans le cas de l'utilisation explicite d'un oracle.

Force est de constater que les mathématiques tributaires de telles démarches informatiques se rapprochent des autres sciences comme la physique qui dépendent de manière plus ou moins essentielle de dispositifs matériels expérimentaux. Ces mathématiques nouvelles sont, dans leur méthode même, d'une nature différente du procédé de la démonstration instauré par les Grecs, qui depuis ce moment inaugural a constitué l'originalité et la marque distinctive de cette discipline. Il est possible que ce soit une nouvelle science qui émerge de cette manière, à la frontière des mathématiques, de la logique et de l'informatique : une science dont les objets sont les démonstrations formelles, et dont la démarche est fortement dépendante d'outils informatiques puissants. La confiance accordée aux résultats fournis par ces démarches peut être très forte. Par exemple, la certification Coq, label donné à un résultat vérifié par l'assistant de preuve Coq, est considérée comme une forme très haute de validité ${ }^{7}$, à tel point que si un résultat obtenu par Coq entrait en contradiction avec un jugement validé par une démonstration au sens développé précédemment de l'évidence vécue, il ne va pas de soi que la confiance serait prioritairement accordée au deuxième. Autrement dit, sur le plan de la force de la confiance, un résultat certifié par Coq n'a rien à envier à un résultat certifié par une démonstration. Mais ce n'est pas la force en elle-même de la confiance accordée à un résultat qui est la marque distinctive de l'essence de la démarche mathématique. C'est la nature du processus qui légitime le résultat, à savoir la démonstration comme expérience de son évidence. Cette expérience crée chez celui qui l'éprouve une forme de rapport particulier avec le résultat validé, rapport qui est incompatible avec la délégation d'une partie de la démonstration à un oracle.

7. Plus précisément, la certification a lieu sur la base d'une inscription de démonstration spécialement adaptée à l'assistant de preuve, élaborée à partir d'une démonstration dont un mathématicien a fait l'expérience et qu'il a rédigée. Alors pourquoi certifier? Trois raisons nous viennent à l'esprit : les erreurs post demonstrationem; convaincre les autres mathématiciens de la correction de la démonstration et en particulier de l'absence d'oubli d'un cas particulier parmi un grand nombre d'autres (théorème de Hales de la conjecture de Kepler, théorème des quatre couleurs) ; développer la certification en tant que discipline scientifique (théorème de FeitThompson; on peut noter qu'au cours de la certification de ce théorème, quelques erreurs de la démonstration originelle ont été corrigées, mais qu'elles étaient superficielles). 


\section{Vers un mode d'existence pour les mathématiques}

Cette partie terminale a pour objet d'introduire un mode d'existence propre aux êtres mathématiques respectant le patron de l'EME et de discuter le placement de ce mode dans celui de la référence [REF]. Préalablement, quelques précisions doivent être apportées relativement au mode [REF] et à la notion générale de mode d'existence au sens de l'EME.

\subsection{Les modes d'existence en général et le mode $[\mathrm{REF}]$ en particulier}

Rappelons que le mode de la référence [REF] est caractérisé par la production de chaines de référence ${ }^{8}$. Une telle chaine se présente extérieurement comme un enchainement d'inscriptions scripto-visuelles. Selon l'EME, ces chaines sont au cœur du mode d'existence [REF], au sens où quelque chose existe objectivement lorsqu'on peut mettre en place une chaine de référence qui établit cette chose via la cascade d'inscriptions constituée par cette chaine. La dissemblance, l'éloignement entre chaque inscription et l'inscription suivante est appelée hiatus. Ce hiatus désigne le manque a priori de conviction que la seconde inscription est conçue de manière à porter une information qu'on peut rapporter à la première, cette conviction étant une expérience recherchée par les acteurs scientifiques qui élaborent la chaine ou en prennent connaissance.

La condition de félicité qui permet de lisser une telle chaine est que l'opération qui fait passer d'une inscription à la suivante soit conçue de manière à préserver cette constance, de telle sorte que l'information lisible sur la deuxième inscription remonte à une information portée par l'inscription précédente. La dernière inscription d'une chaine de référence n'a de valeur que par l'ensemble de la chaine qui, du fait des conditions de félicité, permet de faire transiter cette information en remontant pas à pas la chaine de manière à aboutir au phénomène initial sur lequel porte l'étude.

Il est temps d'énoncer quelques généralités sur les modes d'existence au sens de l'EmE. Un mode d'existence désigne un collectif d'entités partageant une même manière d'être et de s'animer. L'une des idées-phares de l'EME est que, bien qu'une multiplicité de modes d'existence puissent être distingués, ces derniers partagent des traits communs qui permettent de les identifier et de les classer. Ce sont principalement :

- un certain type de trajectoire qui est le support principal de l'existence dans le mode considéré - dans le cas de [REF], ce sont les chaines de référence;

8. Nous renvoyons à la lumineuse étude [Latour 2001] pour un exemple concret de chaine de référence. 
- un certain type de hiatus qui sépare deux étapes successives quelconques d'une trajectoire - dans le cas de [REF], c'est l'éloignement, la dissemblance entre deux inscriptions successives d'une chaine de référence;

- un certain type de conditions de félicité et d'infélicité qui sont les conditions de vérité ou de fausseté propres au mode considéré - dans le cas de $[\mathrm{REF}]$, étant donnée une chaine de référence et une information qui émerge à la dernière étape de la chaine, la condition de félicité énonce que cette information est pertinente si elle peut être rapportée d'étape en étape en remontant toute la chaine de référence.

\subsection{Un mode d'existence pour les mathématiques}

Cette partie suit le patron des modes d'existence pour décrire le fonctionnement, la manière d'être et de se présenter à nous, des êtres qui se manifestent quand un mathématicien met au point ou parcourt un développement mathématique. L'idée est d'exploiter la description empiriste du raisonnement mathématique de la partie 4 .

Préalablement, il convient de revenir sur les inscriptions scripto-visuelles qui interviennent dans la pratique des mathématiques. Dans la partie 4.2, avec la notion d'inscription de démonstration (d'un jugement), nous avons introduit une telle inscription en insistant sur la différence essentielle avec l'expérience de démonstration (du même jugement). Considérons maintenant la notion a priori banale d'énoncé : c'est un texte qui exprime un contenu mathématique, que ce dernier soit établi - ce qui en fait un jugement - ou ne le soit pas. Élargissons cette notion de manière à inclure des éléments non textuels (diagrammes, images, etc.), afin d'en faire un tout complet sur le plan de l'expression habituelle des mathématiques. Après ce préalable, le mode d'existence pour les mathématiques peut être abordé en précisant son type de hiatus, de trajectoire et de condition de félicité.

Le hiatus du mode d'existence pour les mathématiques est la discontinuité manifestée par l'éloignement entre un énoncé donné initialement et un énoncé final. Cet éloignement doit être compris comme l'absence d'expérience de démonstration qui permettrait de passer du premier au second.

Le saut, la transition, la transformation reliant ces énoncés est une inscription de démonstration qui est présentée comme un guide susceptible de faire transiter l'évidence du premier au second. Pour important qu'il soit, ce guide ne certifie pas que l'expérience du cheminement de l'évidence sera au rendez-vous de celui qui le parcourt.

La condition de félicité de ce saut est justement qu'il réussisse à générer l'expérience du cheminement de l'évidence du premier au second énoncé. Lorsque cette condition est réalisée, l'énoncé final par delà le hiatus devient un jugement, sous réserve que l'énoncé source en deçà soit lui-même un jugement, et le saut s'identifie à une inscription de démonstration de ce jugement à partir du jugement source. Une 
chaine démonstrative est une trajectoire obtenue en considérant un enchainement d'énoncés reliés par des sauts. Une telle chaine satisfait la condition de félicité si c'est le cas de chacun de ses sauts.

Un être mathématique (c'est-à-dire un objet ou une propriété mathématique) est amené à l'existence lorsqu'une chaine démonstrative satisfaisant la condition de félicité énonce, montre cette existence. C'est ainsi que tout jugement, habituellement conçu comme un acquis de connaissance, est également interprétable comme une extension de l'ontologie : telle entité (objet ou propriété) est dotée d'une existence avérée par la chaine démonstrative correspondante. Par exemple, une chaine démonstrative associée au théorème de Pythagore amène à l'existence, pour un triangle rectangle, la propriété d'égalité entre les carrés construits sur les côtés adjacents à l'angle droit et le carré construit sur l'hypoténuse.

Dans quel lieu ces êtres vivent-ils? L'ambigüité ontologique des mathématiques relevée par Netz fait qu'aucun lieu n'est attribué d'emblée aux objets mathématiques dans ce que nous nommons naïvement la réalité extérieure. Depuis les mathématiques grecques, la réponse platonicienne à cette difficulté a été de postuler que ces objets gisent dans l'immuable éternité d'un monde suprasensible d'idées pures. Le mode d'existence des êtres mathématiques présenté ici évite ce recours à la transcendance tout en restant au plus près de la pratique des acteurs de cette sciences. Il reste que, si on le souhaite, on peut dire que les entités mathématiques peuplent des mondes qui leur sont propres tout en restant attachées aux dispositifs scripto-visuels qui les supportent.

Comme cela est énoncé dans l'EME, « chaque saut par-dessus une discontinuité représente un risque pris qui peut réussir ou rater ». Dans le cas qui nous intéresse, le risque est que l'inscription de démonstration soit considérée comme insuffisante pour générer l'expérience de l'évidence de l'énoncé final. L'objet de la condition de félicité est donc de surmonter ce risque. Cependant, le risque ne peut pas être éliminé définitivement : c'est la leçon enseignée par le phénomène des erreurs post demonstrationem, c'est-à-dire la nécessité d'envisager l'éventualité d'erreurs dans la démonstration du jugement considéré. Traduite dans notre perspective ontologique, cette leçon montre que l'existence d'un être mathématique ne peut pas avoir la valeur d'une vérité définitive transcendant l'expérience du cheminement de l'évidence éprouvé par un mathématicien. Cet être est doté d'une existence certaine tant que le transport de l'évidence qui le valide est reconnu comme tel, ce qui ne peut exclure la découverte future d'une faille susceptible d'invalider cette existence. La raison en est que ce cheminement est une expérience, celle de l'évidence du jugement final, et non pas l'accès à une forme de réalité indépendante des acteurs mathématiciens. 


\section{3 Éléments de comparaison entre $[\mathrm{REF}]$ et le mode d'existence des êtres mathématiques}

La comparaison entre le mode d'existence des êtres mathématiques et celui de la référence se réduit principalement à la comparaison des chaines et des conditions de félicité propres à chacun. Ceci met en lumière des analogies et des différences entre

- d'une part la technique des chaines de référence pratiquée dans les sciences de terrain ou expérimentales rapidement présentée dans la partie 5.1,

- d'autre part la technique des chaines démonstratives introduite dans la partie 5.2.

La première analogie est que ces deux types de chaines se présentent comme des trajectoires dont les étapes successives sont des inscriptions scripto-visuelles. Une deuxième analogie significative apparait au niveau de la fonction des chaines de référence et des chaines d'existence mathématique. Dans les deux cas, ces dispositifs ont pour but de créer une conviction à propos de la vérité d'un énoncé scientifique. Comme la suite va le montrer, cette ressemblance ne va pas jusqu'à inclure la nature même de cette conviction. Une troisième analogie concerne le hiatus se présentant dans chacun des deux types de chaines, hiatus qui dans les deux cas est formulé de manière identique comme la dissemblance et l'éloignement de deux inscriptions successives. Justement, une quatrième analogie peut être relevée : dans chacune de ces chaines, chaque saut d'une inscription à la suivante doit satisfaire une condition de félicité qui est de préserver certaines constantes. Enfin, une dernière analogie est relative au caractère lointain, c'est-à-dire éloigné des affaires communes, des entités amenées à l'existence selon l'un ou l'autre des deux modes. En effet, il ne faut rien de moins qu'une chaine - de référence ou démonstrative - pour réussir à les instaurer.

Une source notable de dissemblance est relative aux conditions de félicité. En effet, dans le cas d'une chaine démonstrative, cette condition se confond avec l'expérience de l'évidence de la dernière inscription. Cette évidence signifie que l'acteur concerné voit littéralement, perçoit directement, c'est-à-dire sans recours à un oracle, la validité du résultat final. Cette signification de l'évidence est entièrement dépendante de l'aspect diagrammatique de la pensée mathématique. En effet, cette vision directe du résultat final n'est rien d'autre que l'expérience perceptive de diagrammes convenablement (et souvent laborieusement) modifiés. Au contraire, une chaine de référence inclut généralement des transformations - utilisation cruciale d'appareillages technologiques, généralisation inductive du résultat d'expériences singulières - qui ne peuvent pas produire la même forme d'évidence, même si la conviction qui en découle peut elle aussi être très forte.

En guise de conclusion toute provisoire, le mode d'existence des entités mathématiques décrit dans la partie 5.2 et le mode de la référence présentent un nombre significatif d'analogies qui rendent difficiles une séparation pure et simple. Cepen- 
dant, ces deux mêmes modes exhibent aussi au moins une forme de différence assez nette qui empêche l'identification des deux ou même la fusion de l'un dans l'autre. Or ces deux modes semblent nécessaires afin de couvrir le champ scientifique. D'ailleurs, c'est le plus souvent un mixte des deux qui semble être à l'œuvre lorsque la démarche considérée présente à la fois un développement mathématicodéductif et un dispositif expérimental. Un exemple récent et spectaculaire en est l'extraordinaire quête étalée sur près d'un siècle qui vient de confirmer l'existence des trous noirs via la perception des ondes gravitationnelles. La démarche mythique de Thalès déterminant la hauteur d'une pyramide par l'intermédiaire du théorème qui porte son nom est tout aussi exemplaire. À la réflexion, cette idée d'associer les deux modes semble inévitable pour rendre compte de l'activité scientifique même dans le cas des sciences expérimentales ou de terrain. Pour tenir compte de ces contraintes, une solution est de concevoir le mode [REF] sous la forme d'un mode élargi contenant non seulement le mode de la référence restreint décrit dans la partie 5.1 mais aussi le mode d'existence des êtres mathématiques ${ }^{9}$. Si cette hypothèse est retenue, il restera à donner une description cohérente et unifiée de cette acception étendue du mode [REF]. En attendant, il est facile d'esquisser une description schématique de ce mode : ses trajectoires sont des chaines d'inscriptions obtenues chacune à partir de la précédente par une transformation; son hiatus est la dissemblance, l'éloignement des inscriptions successives; sa condition de félicité est la circulation de la référence ou de l'évidence le long de la chaine.

\section{Remerciements}

Les auteurs remercient Isabelle Stengers et Bruno Latour pour l'intérêt qu'ils ont manifesté pour la réalisation de cette étude. Ils remercient aussi Fabien Ferri pour les avoir mis sur la piste des thèses de Peirce commentées dans les écrits [Chauviré 1987], [Chauviré 2008].

Tout en reconnaissant l'originalité et l'intérêt de l'analyse philosophique de la notion de démonstration développée dans le présent texte, un relecteur a fait part de son regret que le mode d'existence des êtres mathématiques qui en découle se cantonne à la seule " preuve d'énoncés déjà donnés » en laissant dans l'ombre la «généalogie des concepts ». De manière générale, il oppose les « gestes à la Châtelet », comme l'invention en Grèce de la ligne sans épaisseur, par lesquels se transforment les paysages conceptuels, à la «monomanie », liée au logicisme, qui réduit les mathématiques à un amoncellement de démonstrations. Nous remercions ce relecteur qui, par cette critique intéressante, nous amène à préciser notre point de vue sur l'importance de l'innovation conceptuelle en mathématiques.

Cette innovation se manifeste dans le travail créatif de la preuve. Un exemple

9. Remarquons que les mathématiques tributaires de l'informatique évoquées dans la partie 4.4 ont un mode d'existence différent des mathématiques habituelles mais relevant clairement de ce mode [REF] élargi. 
emblématique en est donné par les manipulations et enrichissements de diagrammes à l'œuvre dans les constructions auxiliaires de la géométrie des Grecs. D'après l'analyse de Peirce sur la dimension diagrammatique des mathématiques, il s'agit là d'un caractère général et consubstantiel de cette science, valant bien au delà de la seule géométrie. À la suite des travaux de Netz et de Peirce, notre analyse fournit un cadre radicalement éloigné du logicisme et du formalisme pour penser ces actes créatifs et les insérer dans la pratique du travail démonstratif.

L'autre forme d'inventivité conceptuelle est celle qui surgit en amont des preuves, par exemple dans une définition, dans une conjecture, dans le dévoilement d'une analogie entre deux domaines a priori sans rapport, etc. Ces gestes contribuent à modifier les champs d'étude et la compréhension que nous en avons. Pour cela, ils doivent affecter l'activité mathématique par excellence depuis les Grecs qui est le travail d'élaboration des preuves de nouveaux jugements. En accord avec le pragmatisme de Peirce et de James, nous considérons que, in fine, l'importance et le sens d'un tel geste se mesure à ces traces concrètes. Il n'est pas dans l'objectif et la philosophie du présent article d'en interroger le sens lors de l'apparition initiale de ce geste en tant que «stratagème allusif » [Châtelet 1994, p. 221], avant toutes ces conséquences.

L'ontologie des mathématiques que nous avons développée est soucieuse d'une part de la pratique quotidienne dans les lieux où se développent et se communiquent les mathématiques et d'autre part des conditions concrètes, cognitives ou matérielles, de cette pratique. Puisque cette pratique est polarisée par le travail concret sur les démonstrations, nous maintenons qu'il s'agit d'un bon niveau pour juger de l'ontologie de cette science, à condition toutefois de s'appuyer sur la conception empiriste de la démonstration développée dans la partie 4, qui est, faut-il le rappeler, sans rapport avec la caricature logiciste ou formaliste de la preuve. Cette dernière étant la cible explicite de la critique formulée par notre relecteur, nous estimons que notre présent travail n'est pas pleinement concerné par elle, tout en reconnaissant l'intérêt de la démarche qui la porte.

\section{Références}

Bourbaki, Nicolas [1954], Éléments de mathématique : première partie, Les structures fondamentales de l'analyse. Livre I, Théorie des ensembles. Chapitre I, Description de la mathématique formelle. Chapitre II, Théorie des ensembles, Actualités scientifiques et industrielles 1212, Paris : Hermann.

[1970], Éléments de mathématique : Algèbre. Chapitres 1 à 3, Paris : Hermann, nouvelle éd.

Bourdeau, Michel [2004], Présentation : intuitionnisme et philosophie, Re- 
vue internationale de philosophie, 230, 383-400, https://www.cairn.info/ revue-internationale-de-philosophie-2004-4-page-383.htm.

ChÂtelet, Gilles [1994], Le geste bien plutôt que la règle, dans : First European congress of mathematics : Paris, July 6-10, 1992, Vol. III : round tables, Bâle : Birkhäuser, Progr. Math., t. 121, chap. Round table F : Philosophie des mathématiques : pourquoi? comment?, 217-222.

Chauviré, Christiane [1987], Schématisme et analyticité chez C. S. Peirce, Archives de Philosophie, 50(3), 413-437, http://www.jstor.org/stable/ 43035224 .

[2008], L'Eil mathématique, Paris : Éditions Kimé.

DeWEy, John [2014], La Quête de la certitude : une étude de la relation entre connaissance et action, Bibliothèque de philosophie, Paris : Gallimard, traduction et présentation par Patrick Savidan de The quest for certainty : a study of the relation of knowledge and action, 1929.

Esquenazi, Jean-Pierre [1997], Peirce et (la fin) de l'image : sens iconique et sens symbolique, MEI "Médiation Et Information », 6, 59-73, http://www. mei-info.com/revue/6/icone-image.

Gentzen, Gerhard [1955], Recherches sur la déduction logique, Paris : Presses universitaires de France, traduction par Robert Feys et Jean Ladrière de Untersuchungen über das logische Schließen, 1935.

Gonthier, Georges [2005], A computer-checked proof of the Four Colour Theorem, Rap. tech., Microsoft Research Cambridge, https://web.archive.org/ web/20160304224502if_/http://research.microsoft.com/en-US/people/ gonthier/4colproof .pdf.

Husserl, Edmund [1959], Recherches logiques : tome premier : prolégomènes à la logique pure, Épiméthée : essais philosophiques, Paris : Presses universitaires de France, traduction par Hubert Élie de Logische Untersuchungen : erster Band: Prolegomena zur reinen Logik, $2^{\mathrm{e}}$ édition, 1913.

James, William [2005], Essais d'empirisme radical, Marseille: Agone, traduction et présentation par Guillaume Garreta et Mathias Girel de Essays in radical empiricism, 1912.

Kunn, Thomas [1972], La Structure des révolutions scientifiques, Paris : Flammarion, traduction par Laure Meyer de The structure of scientific revolutions, $2^{\mathrm{e}}$ édition, 1970. 
LATOuR, Bruno [1985], Les « vues » de l'esprit : une introduction à l'anthropologie des sciences et techniques, Culture technique, 14, 3-29, http://hdl.handle. net/2042/31688.

[2001], L'Espoir de Pandore : pour une version réaliste de l'activité scientifique, Paris : La Découverte, chap. 2 : Sol amazonien et circulation de la référence, 33-82, traduction par Didier Gille de Pandora's hope : essays on the reality of science studies, 1999.

[2009], Un livre de Reviel Netz pour une approche non formaliste des formalismes, Revue d'anthropologie des connaissances, 3(2), 185-206, doi:10.3917/rac.007.0185, recension de [Netz 1999]. Traduction par Dominique Vinck et Rigas Arvanitis révisée par l'auteur de « The Netz-works of Greek deductions $\gg, 2008$.

[2012], Enquête sur les modes d'existence : une anthropologie des modernes, Paris : La Découverte.

[2013], An inquiry into modes of existence : an anthropology of the moderns, http://modesofexistence.org, Fondation nationale des sciences politiques, Paris.

Lefebvre, Martin [2013], Peirce et l'image : présentation, Recherches sémiotiques, 33, 3-17, doi:10.7202/1035281ar.

Martin-LöF, Per [1987], Truth of a proposition, evidence of a judgement, validity of a proof, Synthese, 73, 407-420, doi:10.1007/BF00484985.

Netz, Reviel [1999], The shaping of deduction in Greek mathematics : a study in cognitive history, Ideas in context, Cambridge : Cambridge University Press.

PeIRCE, Charles Sanders [1933-1958], Collected papers, Cambridge : Harvard University Press, huit tomes.

— [1976], The new elements of mathematics, La Haye : Mouton.

The Coq Development team [1984-2019], The Coq proof assistant reference manual, LogiCal project, http://coq.inria.fr.

Verhaegen, Philippe [1994], Image, diagramme et métaphore : à propos de l'icône chez C. S. Peirce, Recherches en communication, 1, 19-48, http://sites . uclouvain.be/rec/index.php/rec/article/view/291.

Wallet, Guy \& Neuwirth, Stefan [2018], Enquête sur les modes d'existence des êtres mathématiques (version augmentée), https://hal.archives-ouvertes . fr/hal-01824928. 
WeyL, Hermann [1994], Le continu, dans : Le Continu et autres écrits, Paris : Vrin, Mathesis, 33-124, traduction et présentation par Jean Largeault de Das Kontinuum : kritische Untersuchungen über die Grundlagen der Analysis, 1918.

Wittgenstein, Ludwig [2004], Recherches philosophiques, Bibliothèque de philosophie, Paris : Gallimard, traduction par Françoise Dastur, Maurice Élie, JeanLuc Gautero, Dominique Janicaud et Élisabeth Rigal de Philosophische Untersuchungen, 1953.

[2006], De la certitude, Bibliothèque de philosophie, Paris : Gallimard, traduction et présentation par Danièle Moyal-Sharrock de Über Gewißheit, 1969. 